\title{
The effect of stimulus strength on binocular rivalry rate in healthy individuals: Implications for genetic, clinical and individual differences studies
}

\author{
Phillip C.F. Law ${ }^{\mathrm{a}, *}$, Steven M. Miller ${ }^{\mathrm{a}, \mathrm{b}}$, Trung T. $\mathrm{Ngo}^{\mathrm{a}, \mathrm{c}, \mathrm{d}}$ \\ ${ }^{a}$ Monash Alfred Psychiatry Research Centre, Monash University Central Clinical School and The Alfred Hospital, Melbourne, Australia \\ b School of Psychological Sciences, Monash University, Melbourne, Australia \\ c Genetic Epidemiology Laboratory, QIMR Berghofer Medical Research Institute, Brisbane, Australia \\ d Mater Research Institute-UQ, Neurosciences \& Cognitive Health Program, Faculty of Medicine, University of Queensland, Brisbane, Australia
}

\section{A R T I C L E I N F O}

\section{Keywords:}

Binocular rivalry rate endophenotype

Mixed percepts

Stimulus strength

Drift speed

Aperture size

Individual differences

Levelt

\begin{abstract}
A B S T R A C T
Binocular rivalry (BR) occurs when conflicting images concurrently presented to corresponding retinal locations of each eye stochastically alternate in perception. Anomalies of BR rate have been examined in a range of clinical psychiatric conditions. In particular, slow BR rate has been proposed as an endophenotype for bipolar disorder (BD) to improve power in large-scale genome-wide association studies. Examining the validity of BR rate as a BD endophenotype however requires large-scale datasets ( $n=1000 \mathrm{~s}$ to $10,000 \mathrm{~s}$ ), a standardized testing protocol, and optimization of stimulus parameters to maximize separation between BD and healthy groups. Such requirements are indeed relevant to all clinical psychiatric BR studies. Here we address the issue of stimulus optimization by examining the effect of stimulus parameter variation on BR rate and mixed-percept duration (MPD) in healthy individuals. We aimed to identify the stimulus parameters that induced the fastest BR rates with the least MPD. Employing a repeated-measures within-subjects design, 40 healthy adults completed four BR tasks using orthogonally drifting grating stimuli that varied in drift speed and aperture size. Pairwise comparisons were performed to determine modulation of BR rate and MPD by these stimulus parameters, and individual variation of such modulation was also assessed. From amongst the stimulus parameters examined, we found that 8 cycles/s drift speed in a $1.5^{\circ}$ aperture induced the fastest BR rate without increasing MPD, but that BR rate with this stimulus configuration was not substantially different to BR rate with stimulus parameters we have used in previous studies (i.e., 4 cycles/s drift speed in a $1.5^{\circ}$ aperture). In addition to contributing to stimulus optimization issues, the findings have implications for Levelt's Proposition IV of binocular rivalry dynamics and individual differences in such dynamics.
\end{abstract}

\section{Introduction}

Binocular rivalry (BR) is an intriguing visual phenomenon in which conflicting images presented to each eye are perceived in alternation rather than being superimposed. For example, simultaneously presenting a vertical grating to one eye, and a horizontal grating to the other eye, induces perception of the vertical grating for a few seconds, followed by perception of the horizontal grating for a few seconds, and so on (Fig. 1). BR and other perceptual rivalry types such as ambiguous figures have previously been examined, particularly with respect to alternation rate, in the context of clinical psychiatric disorders from the early to mid-20th Century (e.g., $[14,17,20,21,25,27,37,51,58,74])$ ). The modern clinical focus on BR emerged with reports from Australia that
$\mathrm{BR}$ rate was slow in the heritable psychiatric condition, bipolar disorder (BD), relative to healthy individuals (e.g., $[53,68]$ ) — a finding that has since been independently replicated in populations from Japan [57], New Zealand [82] and China [87].

Following Pettigrew and Miller's [68] original study on BD, other clinical psychiatric conditions have been examined including schizophrenia and major depression [39,53], autism spectrum conditions [4,26,42,71-73], attention deficit hyperactivity disorder (e.g., [3,6]), and generalized social anxiety disorder [5]. Although some researchers (e.g., [82]) have attempted to use the same testing protocol as that of Pettigrew and Miller [68], so that data may be directly compared between clinical studies, other researchers have employed different test protocols (e.g., shorter viewing durations, different stimulus characteristics, different

\footnotetext{
* Corresponding author at: Level 4, 607 St Kilda Road, Melbourne, VIC 3004, Australia.

E-mail address: Phillip.Law@monash.edu (P.C.F. Law).
} 
PRESENTED STIMULI

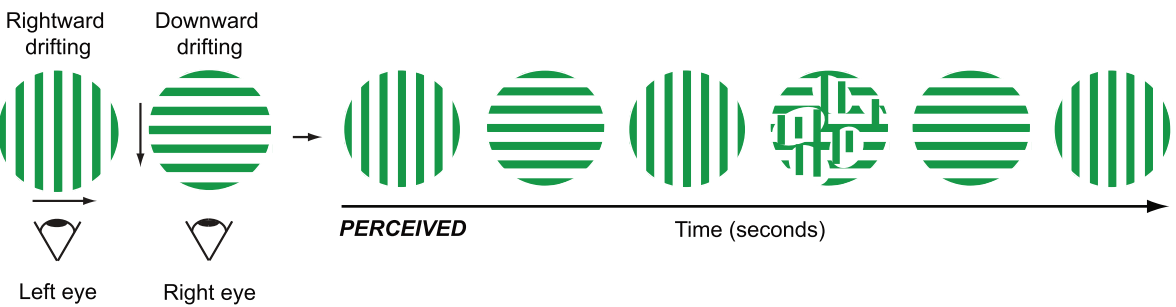

Fig. 1. Binocular rivalry. Presenting dissimilar images simultaneously — such as rightward-drifting vertical gratings and downward-drifting horizontal gratings - one to each eye (i.e., dichoptic presentation), causes each image to stochastically alternate in perception. Mixed or piecemeal percepts (i.e., portions of both eyes' presented images are simultaneously visible) occur occasionally during the transition between perception of the presented images. Arrows adjacent to the presented stimuli denote the direction of grating drift. response options), making comparisons difficult. Such issues become particularly relevant when considering potential applications of BR findings in genetic studies of clinical psychiatric disorders.

Pettigrew and Miller [68] and Miller et al. [53] demonstrated high sensitivity and reliability of the BR rate trait in BD. This earlier work was followed by a large twin study demonstrating high heritability of the trait and confirming its high reliability ([54]; see also [76]). This heritability study supported the original proposal [68] that slow BR could be used as an endophenotype for BD (reviewed in $[59,60]$ ). Endophenotypes - or intermediate phenotypes - can enhance power in gene-finding studies of complex psychiatric diseases by using the relevant quantitative trait to classify a genotype as affected rather than manifestation of the clinical disorder (see [30,31,33,43]). However, such application requires large-scale studies of thousands to tens of thousands of subjects (see $[24,36,45,50,86]$ ). Elsewhere we have discussed prospects for an online platform of BR testing to address these large sample-size requirements [46]. Such a platform not only facilitates the collection of very large sample-sizes, but also enables the prospect of standardized BR testing across clinical conditions and research centres, for purposes of direct comparison between clinical studies.

For any such endeavor striving for large-scale, standardized BR testing in clinical conditions, the optimal stimulus parameters also require examination. Changing stimulus parameters can change the signal strength of the stimulus or its stimulus strength, which can in turn modulate BR rate. For example, higher contrast, faster drift speed, and brighter luminance are all considered to induce greater stimulus strength (see below). However, the sensitivity function of stimulus-strength rate modulations is not always monotonic (e.g., [44]). In the study by Pettigrew and Miller [68], a high-strength stimulus (i.e., orthogonally drifting gratings of high spatial frequency; 8 cycles $/{ }^{\circ}$ ) induced significantly slower BR rate in a group of euthymic subjects with BD relative to healthy controls, with wide group separation. The finding was independently replicated using the same high-strength stimulus [82] and using an intermediate-strength stimulus [57]. Following Pettigrew and Miller's [68] original study, a subsequent study by Miller et al. [53] using a low-strength stimulus (i.e., stationary gratings of lower spatial frequency; 4 cycles $/^{\circ}$ ) also demonstrated significantly slower BR rate in BD than in healthy individuals, though with less evident group separation. Comparing the data in these two studies (i.e., $[53,68]$ ) suggested that the greater group separation in the earlier study may have been due to the high-strength stimuli producing a faster average BR rate in healthy individuals, while BD subjects remained robustly slow whether viewing high- or low-strength stimuli. On this interpretation, BD subjects would be relatively insensitive to stimulus-related BR rate modulation compared with healthy individuals (discussed in [53]; see also [59]), and therefore viewing of higher-strength stimuli should maximize group separation. However, this comparison between the data of Miller et al. [53] and Pettigrew and Miller [68] is limited by the fact that control subjects were different between the two studies, as were the BD subjects. What is needed to directly assess the hypothesis that individuals with $\mathrm{BD}$ have robustly slow $\mathrm{BR}$ rates (i.e., relatively insensitive to stimulus-related BR rate modulation) is varying stimulus strength in the same $\mathrm{BD}$ and control subjects (i.e., a within-subject design).

Here we report a within-subject study in healthy individuals that aims to determine whether viewing higher-strength stimuli - using grating drift speed as the stimulus strength factor - can induce faster $\mathrm{BR}$. The predominance of drifting gratings over stationary gratings increases with drift speed ([83]), suggesting that changing from stationary to drifting stimuli increases stimulus strength (in accordance with [48]; see below). It is not clear, however, whether the sensitivity function for drift speed is non-monotonic and whether gratings drifting at 4 cycles/s as used in previous studies $[54,68,82]$ are the peak of such a non-monotonic function. Hence 8 cycles/s gratings are also assessed in the current study to examine whether this particular drift speed drives BR rate faster than 4 cycles/s gratings, or whether the 4 cycles/s gratings represent a ceiling effect for BR rate. Here we report a comparatively large within-subject BR dataset of healthy individuals ( $n=40)$ to directly assess and clarify the effect of stimulus strength on $\mathrm{BR}$ rate.

The study protocol also enabled assessment of a secondary aim, i.e., the effect of stimulus size on mixed-percept duration (MPD). MPD is the total time spent perceiving mixed percepts in a given BR viewing period, and provides a measure of the degree of perceptual mixing between each eye's presented image. BR rate is derived by dividing the total number of perceptual alternations by the total BR viewing period, excluding responses to mixed percepts. As such, reducing an individual's total MPD provides more data on which to base the calculation of $\mathrm{BR}$ rate and thus improves accuracy of the BR rate measure. There have been reports that smaller BR stimuli between $0.5^{\circ}$ and $2^{\circ}$ of visual angle increase exclusive percept visibility ([63]; see also $[8,78]$ ), which corresponds to a shorter MPD. The current study thus aimed to examine whether reducing the size of a BR stimulus from $1.5^{\circ}[53,54]$ to $1^{\circ}$ or $0.5^{\circ}$ of visual angle would produce a shorter MPD. We did not assess stimuli subtending larger than $1.5^{\circ}$ so as to avoid inducing a longer MPD. Furthermore, because earlier studies examining the effect of stimulus size on exclusive visibility used only small samples ([8,64,78]; $n=3$ and 4 and 11, respectively), the current study employed a comparatively large dataset $(n=40)$ to clarify the effect of stimulus size modulation on MPD. However, interpretation of these MPD data will require caution as the mixed-percept response option also included subjects' erroneous responses (see Methods and Discussion).

The current experiment is also relevant to the historical literature because stimulus-related modulation of BR temporal dynamics has been a focus for rivalry researchers since Breese [12] (see also Wade \& Ngo, [84]) and especially since the seminal four-proposition framework of BR dynamics by Levelt [48]. Recently reviewed in detail by Brascamp et al. [11], these propositions have mostly been examined experimentally by assessing contrast-modulated dominance duration (i.e., the time a percept maintains exclusive dominance). Such experiments involve keeping constant the stimulus strength presented to one eye, while manipulating the stimulus strength presented to the other eye (see Levelt's Proposition III discussed in [11]). Relevant to the current study, Levelt's Proposition IV holds that increasing the stimulus strength matched between both eyes should induce a faster BR rate, and this has indeed been observed using dominance duration as the dependent variable and contrast as the stimulus strength factor (e.g., $[2,12,13,19,69])$. Moreover, two earlier reports indicated that 
increasing stimulus strength matched between both eyes up to a certain level - where spatial frequency was the stimulus strength factor produced more BR alternations in a given observation period, but the number of alternations decreased beyond that level $[44,64]$. There has also been mention in the literature, based only on unanalysed data and limited pilot observations, of greater stimulus strength (using drift speed) presented to both eyes inducing a faster BR rate [61]. Other than these pilot observations however, to our knowledge no study has yet properly examined Levelt's Proposition IV using drift speed as the stimulus strength factor. The experimental protocol of the current study thus enabled direct testing of Levelt's Proposition IV with this stimulus strength factor, albeit within a restricted range of drift speeds.

Finally, compared with typical psychophysics experiments, the relatively large sample size in the current study enables, for the first time, assessment of individual differences in stimulus-related modulation of BR rate. The issue of individual differences in psychophysical and visual functions has been a topic of resurgent interest and enables new means of probing genetic and environmental influences on sensory and perceptual systems, as well as neurobiological and pathophysiological mechanisms underlying such influence (e.g., [15,32,40,41,49,65,67,85]).

\section{Methods}

\subsection{Participants}

Forty naïve healthy adults aged between 20 and 66 years (mean age $=34.4 \pm 12.7$ years; 21 males) with normal or corrected-to-normal vision (6/9 or better in both eyes) participated in the study. Written, informed consent was obtained in the presence of a witness prior to testing according to a protocol approved by the Alfred Human Research Ethics Committee and Monash University Human Research Ethics Committee. The research was conducted in accordance with the Declaration of Helsinki. Visual acuity was assessed with a Snellen chart from a distance of $3 \mathrm{~m}$. Reduced visual acuity decreases an individual's perceived contrast and spatial frequency of the stimulus and thus reduces BR rate ([22]; see also [35]). Handedness was assessed using the Edinburgh Handedness Inventory [62]. All participants had their medical and psychiatric history screened using a brief questionnaire and the Mini International Neuropsychiatric Interview [77] to exclude individuals with a psychiatric disorder (e.g., BD, schizophrenia, major depressive disorder), neurological disorder (e.g., epilepsy), brain injury, or visual disorders (e.g., strabismus, amblyopia, color vision deficiency). Subjects were also screened to exclude individuals with first-degree relatives diagnosed with a psychiatric disorder.

State, trait, and clinical ratings were examined along with psychometric measures prior to the testing session for all subjects. Trait and state anxiety were assessed with the State-Trait Anxiety Inventory (STAI; [79]; mean $=33.50 \pm 8.59$ and $24.25 \pm 8.44$, respectively). Depressive state was assessed with the Montgomery-Åsberg Depression Rating Scale (MÅDRS; [55]; mean = $1.48 \pm 2.61$ ). Subjective mood was assessed with a 10-point self-report visual analogue scale $(1=$ 'the worst you have ever felt' to $10=$ 'the best you have ever felt'; mean $=7.45 \pm 1.24$ )

\subsection{Study protocol}

Participants abstained from consuming caffeinated drinks, tobacco, and alcohol for $4 \mathrm{~h}$ prior to testing given their known effects on BR rate $[7,18,28,52,75]$. All participants completed BR tasks under the supervision of an experimenter throughout the testing session to ensure task compliance (see Section 2.3). The BR measures reported in the current study were obtained along with eye-movement task measures. The eyemovement tasks were completed separately and counterbalanced with the BR tasks across participants to avoid potential order effects. Analyses presented in the current study relate only to the BR data. The eyemovement data providing evidence for no relationship with $\mathrm{BR}$ rate are reported elsewhere [47].

\subsection{Binocular rivalry task: apparatus and experimental protocol}

BR stimuli were generated with custom software programmed using Psychtoolbox-3 $[10,66]$ in conjunction with MATLAB ${ }^{\text {TM }}$ (MathWorks Inc., Natick, MA, USA). The specific square-wave stimuli were green rightward-drifting vertical and downward-drifting horizontal gratings. The stimuli had a spatial frequency of 5.33 cycles $/{ }^{\circ}$, were isoluminant between the two eyes, and were presented in a circular aperture on a black background (stimulus contrast $=0.99$ ). Drift speed was either 4 or 8 cycles $/ \mathrm{s}$. The luminance of all stimuli (mean $=4.8 \mathrm{~cd} / \mathrm{m}^{2}$ ) and the background $\left(0.35 \mathrm{~cd} / \mathrm{m}^{2}\right)$ was measured using a LS-100 luminance meter (Konica Minolta Sensing Americas Inc., Ramsey, NJ, USA) through passive polarizer filters. The four BR stimulus conditions were: (i) 4 cycles/s drift speed in an aperture subtending $1.5^{\circ}$ of visual angle; (ii) 8 cycles/s drift speed in an aperture subtending $0.5^{\circ}$ of visual angle; (iii) 8 cycles/s drift speed in an aperture subtending $1^{\circ}$ of visual angle; and (iv) 8 cycles/s drift speed in an aperture subtending $1.5^{\circ}$ of visual angle.

Subjects were instructed to blink naturally and record what they observed passively (i.e., not to preferentially respond to any of the percepts or try to influence their perceptions). Subjects pressed one raised key $(\mathrm{V})$ on a standard keyboard in response to the left eye's presented image, and an adjacent raised key (B) in response to the right eye's presented image. A third response option (spacebar) was used to indicate response error or the perception of either mixed (e.g., checkerboard or mosaic image) or unusual percepts (e.g., filled circle or double images). BR testing was conducted in a quiet, dimly illuminated room. BR behavioral data collection was run with custom software generated in MATLAB $^{\text {TM }}$ (MathWorks Inc., Natick, MA, USA) for Windows $7^{\mathrm{TM}}$ on the customized PC (see below).

After familiarizing subjects with the BR task, the BR testing session comprised five 7-min blocks (see Fig. 2), each comprising four 100-s trials. The blocks were separated by 110-s rest breaks and the trials 30-s rest breaks. The first few minutes of BR viewing have been characterized by increases in BR rate within individuals [1,16,29,34,35,80]. However, BR rates stabilize with longer BR viewing periods [53,54], yielding a more accurate measure of an individual's BR rate. Therefore, the first block served to adequately stabilize BR rates for the remaining

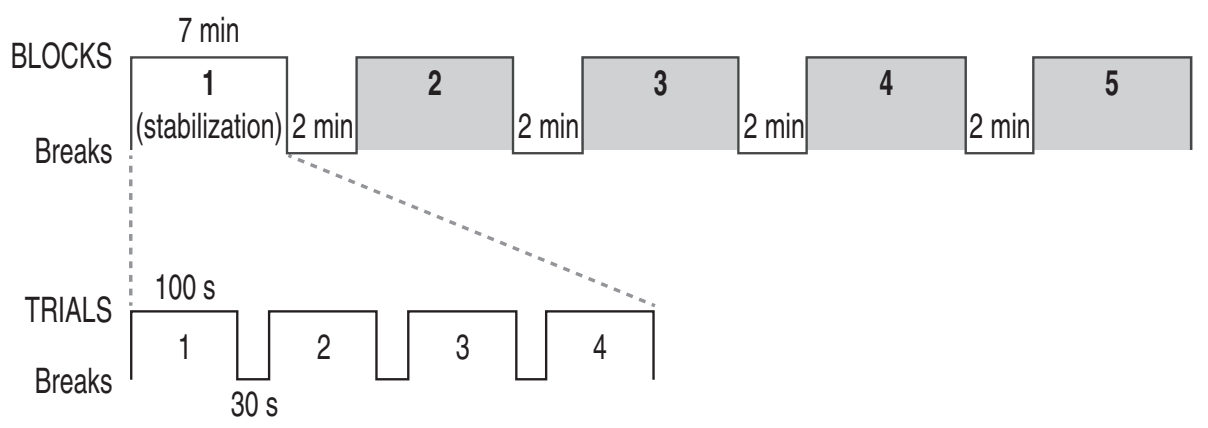

Fig. 2. Binocular rivalry testing protocol. Each block comprised $7 \mathrm{~min}$ of rivalry viewing across four 100-s trials, with rest breaks interspersed between the blocks and trials ( $2 \mathrm{~min}$ and $30 \mathrm{~s}$, respectively). Each of the 40 subjects completed all four stimulus conditions (grey blocks). Four subgroups $(n=10)$ were each run on a different stimulus condition in blocks $1-2$, followed by the remaining (respective) three stimulus conditions in test blocks 3-5 in counterbalanced order across subjects within each subgroup. Therefore, each of the 40 participants completed all four BR stimulus conditions. 
four test blocks and familiarize the subject with the task to diminish the effects of any response errors. To avoid potential order effects, the four BR stimulus conditions were counterbalanced across four subgroups of subjects ( $n=10$ each). Each subgroup was run on a different BR stimulus condition for Blocks 1-2. For Blocks 3-5, participants within each subgroup completed the remaining (respective) three BR stimulus conditions, which were counterbalanced across participants within the subgroup. Therefore, each of the 40 participants completed all four BR stimulus conditions.

All BR stimuli were dichoptically presented on a specialized 19-inch dual-screen liquid crystal display monitor (True3Di $^{\mathrm{Tm}}$; Sharper Technology Inc., Palo Alto, CA, USA; $60 \mathrm{~Hz}$ frame rate, $1280 \times 1024$ pixel resolution). Each screen was directly behind one of two linear polarizers oriented at right angles to each other, and a half-silvered mirror (beam-combiner) oriented at a $45^{\circ}$ angle was between the polarizers. To induce BR, conflicting images of a BR stimulus were independently and simultaneously presented at corresponding central positions on separate screens that projected each image in orthogonal planes (angles) of polarization. One image is transmitted through the half-silvered mirror while the adjacent image is reflected off the mirror, resulting in an interleaved (superimposed) stimulus of two orthogonally polarized images when naturally viewed (see [46]). Subjects viewed the polarized stimulus through passive linear polarizer filters at eye level from a distance of $3 \mathrm{~m}$, resulting in the presentation of conflicting images to corresponding retinal locations of both eyes. Each polarizer filter was tuned to a distinct plane of polarization that enabled the exclusive presentation of one image to one eye while blocking its presentation to the other eye. The result is that simultaneously, the left eye always viewed vertical gratings and the right eye always viewed horizontal gratings. The True3 $\mathrm{Di}^{\mathrm{TM}}$ monitor used to present $\mathrm{BR}$ stimuli was connected to a customized PC (Vostro 460 mini-tower; Dell Inc., Round Rock, TX, USA). This PC was fitted with a Gigabyte ${ }^{\mathrm{TM}}$ ATI Radeon HD 6850 video card, 8GB RAM, and Cooler Master ${ }^{\mathrm{TM}}$ eXtreme Power Plus $700 \mathrm{~W}$ power supply unit. These modifications were to enable adequate processing capacity by the PC as it was concurrently connected to both the True3Di ${ }^{\mathrm{TM}}$ monitor for BR stimuli presentation and a 24-inch singlescreen liquid crystal display monitor (P2412H; Dell Inc., Round Rock, TX, USA; $60 \mathrm{~Hz}$ frame rate, $1280 \times 1024$ pixel resolution) for displaying the trial-based BR data collection protocol.

The passive linear polarizer method for dichoptic viewing has negligible crosstalk and, when viewed with the head in neutral position, there is minimal ghosting (i.e., the subjective perceptual consequence of crosstalk, whereby there is faint perception in one eye of the other eye's intended image; see [46]). To ensure BR viewing was not influenced by the effects of ghosting, subjects were instructed to (i) not tilt or rotate their head, and (ii) view the BR stimulus through the centre of the polarizer filters.

\subsection{Data analysis}

Analysis of participants' BR data employed custom software developed in MATLAB ${ }^{\mathrm{TM}}$ (MathWorks Inc., Natick, MA, USA). BR rate was calculated by dividing the total number of perceptual alternations by the total time of $\mathrm{BR}$ viewing (expressed in $\mathrm{Hz}$ ), excluding mixed or unusual percepts and erroneous responses (i.e., incorrectly pressed key responses) which were indicated by pressing the spacebar. Along with BR rate, MPD was assessed, however MPD is only an approximation of the total time spent perceiving mixed percepts because the spacebar response was also used to indicate response error and unusual percepts. Pressing of the spacebar not only initiated onset of a recorded MPD interval, it was also designated by the data analysis program to disregard the immediately previous recorded response to a perceived image (in case the spacebar had been pressed to indicate a previously erroneous response). Notwithstanding the necessary cautious interpretation due to the conflation of MPD with response errors, in a given observation period, a relatively short MPD corresponds to a relatively greater amount of data being collected for calculating BR rate, thus reflecting a more representative and accurate measure of an individual's true $\mathrm{BR}$ rate.

Predominance is the prevailing dominance of one image over the other in a given observation period, and was calculated by dividing the total time spent perceiving the vertical grating by the total time spent perceiving the horizontal grating (in seconds). The resulting ratio value was log-transformed $\left(\mathrm{PR}_{\log }\right)$ to account for the disproportionate numerical representation in predominance (i.e., any value $>1$ for one image cf. values between 0 and 1 for the other image). As such, where there is no perceptual predominance, $\mathrm{PR}_{\log }$ equals zero, whereas $\mathrm{PR}_{\log }$ values less than zero or greater than zero indicate a perceptual predilection towards the horizontal grating or vertical grating, respectively. Individuals' $\mathrm{BR}$ rate, total $\mathrm{MPD}$, and $\mathrm{PR}_{\mathrm{log}}$ were calculated for each trial. For each individual, the mean BR rate, total MPD, and mean $\mathrm{PR}_{\log }$ were calculated for all trials. The stabilization block was excluded from analysis. Statistical analyses were performed with PASW Statistics 17 and R (version 3.2.5; [70]).

\section{Results}

\subsection{Stimulus-strength modulation of binocular rivalry rate}

$\mathrm{BR}$ rate was compared between the stimulus conditions to examine stimulus-strength modulation effects. Normality was violated for the distributions of BR rate (Shapiro-Wilk test; $p<0.05$ ). A Friedman test with $\mathrm{BR}$ rate as the dependent variable and stimulus conditions as the independent variable showed a significant difference in BR rate across the stimulus conditions $\left(p=3.40 \times 10^{-10}\right)$. Pairwise comparisons

Table 1

Binocular rivalry (BR) rate, mixed-percept duration (MPD) and log-transformed predominance ratio ( $\mathrm{PR}_{\log }$ ) for all stimulus conditions.

\begin{tabular}{llll}
\hline & & Median \pm MAD \\
\hline & $4 \mathrm{c} / \mathrm{s}, 1.5^{\circ}$ & $8 \mathrm{c} / \mathrm{s}, 1.5^{\circ}$ & $8 \mathrm{c} / \mathrm{s}, 1^{\circ}$ \\
\hline BR rate (Hz) & $0.47 \pm 0.12$ & $0.52 \pm 0.10$ & $0.48 \pm 0.11$ \\
MPD (s) & $62.81 \pm 41.17$ & $60.23 \pm 35.41$ & $71.37 \pm 33.46$ \\
$\mathrm{PR}_{\log }$ & $0.09 \pm 0.12$ & $0.09 \pm 0.17$ & $0.40 \pm 0.11$ \\
\hline
\end{tabular}

Mean $\pm S D$

\begin{tabular}{llll}
\hline & $4 \mathrm{c} / \mathrm{s}, 1.5^{\circ}$ & $8 \mathrm{c} / \mathrm{s}, 1.5^{\circ}$ & $8 \mathrm{c} / \mathrm{s}, 1^{\circ}$ \\
\hline BR rate (Hz) & $0.53 \pm 0.22$ & $0.57 \pm 0.24$ & $0.54 \pm 0.23$ \\
MPD (s) & $72.12 \pm 53.09$ & $74.34 \pm 69.35$ & $0.43 \pm 0.16$ \\
PR & $0.08 \pm 0.23$ & $0.13 \pm 0.28$ & $0.03 \pm 73.98$ \\
log & & $0.01 \pm 0.31$ & $0.14 \pm 0.45$ \\
\hline
\end{tabular}

c/s: cycles/s. ${ }^{\circ}$ : degrees of visual angle in a circular aperture. MAD: median absolute deviation. SD: standard deviation. Hz: hertz. s: seconds. 
(a)

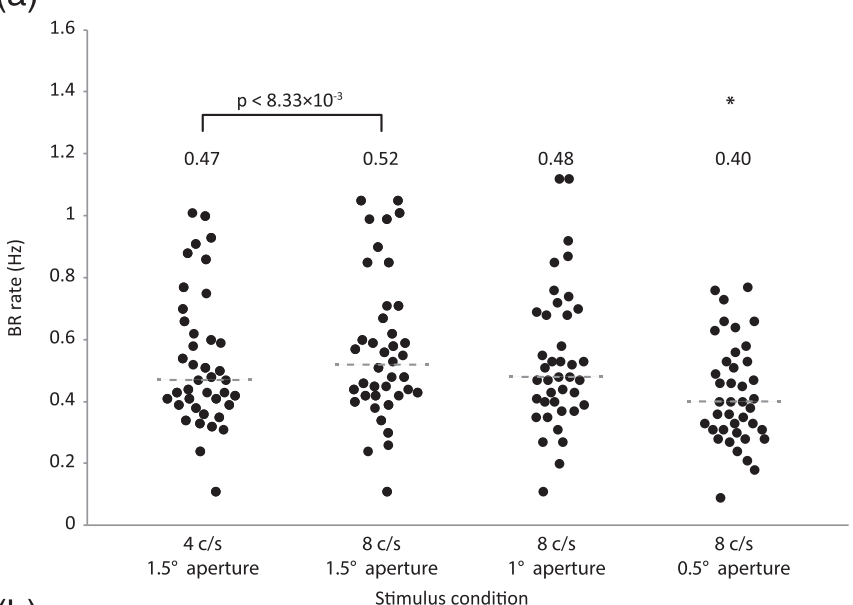

(b)

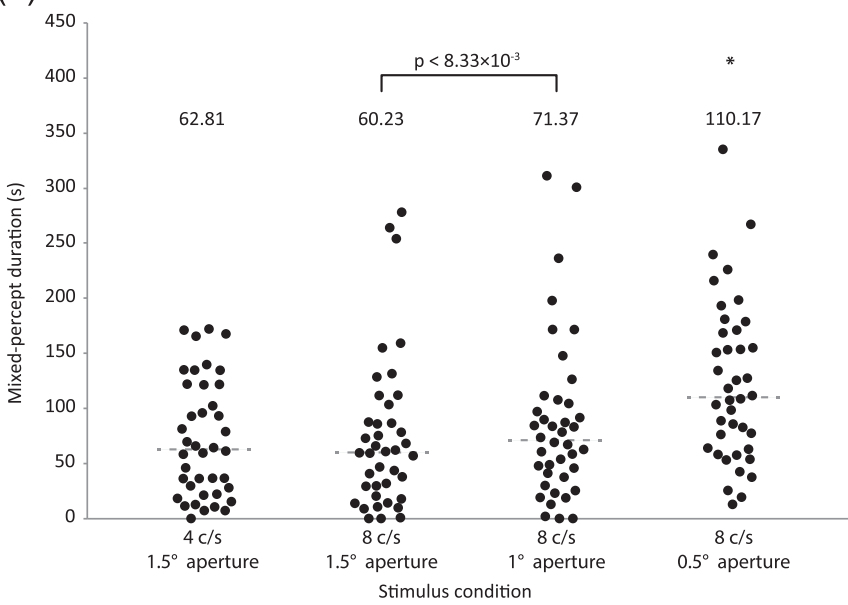

Fig. 3. Column scatter plots showing (a) binocular rivalry (BR) rate and (b) mixed-percept duration (MPD) for the four stimulus conditions. Each solid black dot on the scatter plots represents an individual data point within the respective stimulus condition. Dashed horizontal lines denote the group median value (in accordance with non-parametric statistics) with the numerical value shown above each line. Horizontal brackets above a pair of column scatter plots denote a significant statistical difference between the two stimulus conditions for the corresponding BR measure, $p<8.33 \times 10^{-3}$ (Bonferroniadjusted $\alpha$ of $0.05 / 6$ Wilcoxon signed rank tests). * denotes a significant statistical difference between a particular stimulus condition and all the other stimulus conditions in the respective scatter plot, $p<8.33 \times 10^{-3}$ (Bonferroni-adjusted $\alpha$ of $0.05 / 6$ Wilcoxon signed rank tests). s: seconds. c/s: cycles/s. Stimuli drifting at $8 \mathrm{c} / \mathrm{s}$ in a $1.5^{\circ}$ aperture produced the fastest BR rate and the shortest MPD (see Discussion).

showed that BR rate for a $1.5^{\circ}$ aperture stimulus was significantly faster at 8 cycles/s than at 4 cycles/s $\left(p=3.43 \times 10^{-3}\right.$; Bonferroni-adjusted $\alpha$ : $0.05 / 6$ Wilcoxon signed rank tests $=8.33 \times 10^{-3}$; see Table 1 and Fig. 3a). In contrast, BR rate for a $0.5^{\circ}$ stimulus was significantly slower compared with $1^{\circ}$ and $1.5^{\circ}$ stimuli drifting at 8 cycles $/ \mathrm{s}$ $\left(p=3.70 \times 10^{-6}\right)$, and compared with a $1.5^{\circ}$ stimulus drifting at 4 cycles/s $\left(p=1.32 \times 10^{-5}\right)$. There was no significant difference in BR rate between a $1^{\circ}$ aperture stimulus drifting at 8 cycles $/ \mathrm{s}$ and a $1.5^{\circ}$ stimulus drifting at 4 or 8 cycles/s $\left(p \geq 5.91 \times 10^{-2}\right)$. The results for these comparisons remained non-significant at a less conservative $\alpha$ of 0.05 .

\subsection{Individual variation in stimulus-strength modulation of binocular rivalry rate}

To examine the individual variation in stimulus-strength modulation of BR rate, an individual's ratio of BR rates (r-BR) was calculated by dividing BR rate for 8 cycles/s by that for 4 cycles/s (in a $1.5^{\circ}$ aperture). The resulting value was $\log$-transformed $\left(\mathrm{r}-\mathrm{BR}_{\log }\right)$ to account for the

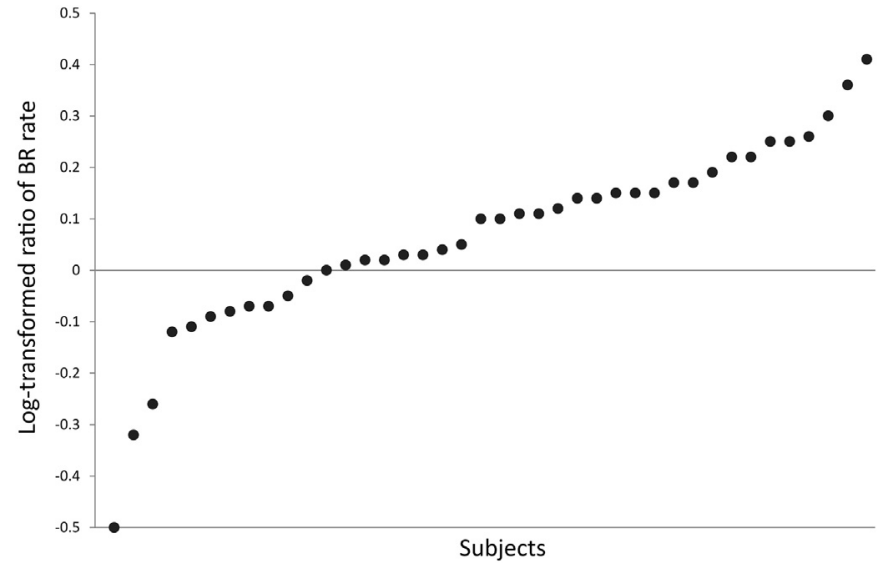

Fig. 4. Individual variation in stimulus-strength modulation of binocular rivalry (BR) rate. The log-transformed ratio of $\mathrm{BR}$ rates $\left(\mathrm{r}-\mathrm{BR}_{\log }\right)$ is indicated on the ordinate $(y$ axis), with each data point of $r-B_{\log }$ presented in ascending order on the abscissa ( $x$ axis). $\mathrm{r}$ $\mathrm{BR}_{\log }$ is calculated by dividing $\mathrm{BR}$ rate for 8 cycles/s by that for 4 cycles/s (in a $1.5^{\circ}$ aperture stimulus) and log-transforming the resulting ratio value. A $r-\mathrm{BR}_{\log }$ value of zero denotes no stimulus-strength modulation of $\mathrm{BR}$ rate, whereas $\mathrm{r}-\mathrm{BR}_{\log }$ values less than zero or greater than zero denote a slower or faster BR rate with greater stimulus strength, respectively. Increasing stimulus strength, through increasing the drift speed from 4 to 8 cycles/s in a $1.5^{\circ}$ aperture stimulus, produced a faster $\mathrm{BR}$ rate (i.e., a r-BR $\mathrm{BR}_{\log }>0$; Levelt's Proposition IV) in a majority of healthy individuals ( $n=28$ or $70 \%)$. However, several individuals ( $n=11$ or $27.5 \%$ ) exhibited a reverse effect, i.e., a slower BR rate as drift speed increased (i.e., $r-B_{\log }<0$ ), and one individual showed no modulation of $\mathrm{BR}$ rate $\left(\mathrm{r}-\mathrm{BR}_{\log }=0\right)$.

disproportionate numerical representation in BR rate, i.e., any value $>1$ for one direction of stimulus-strength modulation whereas values are between 0 and 1 for the other direction. As such, where there is no stimulus-strength modulation of an individual's $B R$ rate, $r-B_{\log }$ equals zero, whereas $r-\mathrm{BR}_{\log }$ less than zero or greater than zero indicates a slower or faster BR rate with faster drift speed, respectively. Increasing drift speed from 4 to 8 cycles $/ \mathrm{s}$ in a $1.5^{\circ}$ aperture was found to induce a faster BR rate in a majority of individuals $\left(\mathrm{r}-\mathrm{BR}_{\log }>0\right.$ in $70 \%$ of subjects; see Fig. 4). However, it is also evident that several individuals showed exactly the reverse effect of a slower BR rate as drift speed increased ( $\mathrm{r}-\mathrm{BR}_{\log }<0$ in $27.5 \%$ of subjects), while one individual showed no modulation of $B R$ rate $\left(r-B R_{\log }=0\right)$.

\subsection{Covariance of binocular rivalry rate between stimuli}

Spearman's $\rho$ correlations were performed to assess the similarity (or covariance) of individuals' BR rates between all stimulus conditions. There was a pattern of significant, high positive correlations in BR rate between all stimulus conditions $\left(\rho=0.79-0.91, p \leq 1.04 \times 10^{-9}\right.$, one-tailed; Bonferroni-adjusted $\alpha: 0.05 / 6$ Spearman's $\rho$ tests $=8.33 \times 10^{-3}$ ) - indicating that individuals' BR rates strongly covaried across the stimulus conditions. In addition, a significant high intraclass correlation indicated low variance (or high clustering) in BR rate within each stimulus condition $\left(r=0.95, p=8.70 \times 10^{-37}\right.$; average measures, two-way mixed model). These findings indicate that the observed differences in BR rate between the stimulus conditions can be attributed to the manipulation of stimulus strength factors.

\subsection{Stimulus-strength modulation of mixed-percept duration}

MPD was compared between the stimulus conditions to examine stimulus-strength modulation effects on this BR measure. Normality was violated for the distributions of MPD (Shapiro-Wilk test; $p<0.05$ ). A Friedman test with MPD as the dependent variable and stimulus conditions as the independent variable showed a significant difference in MPD across the stimulus conditions $\left(p=8.61 \times 10^{-6}\right)$. Pairwise comparisons showed that MPD for an 8 cycles/s stimulus was significantly shorter in a 
Table 2

Spearman's $\rho$ between binocular rivalry (BR) rate, mixed-percept duration (MPD), and log-transformed predominance ratio ( $\mathrm{PR}_{\mathrm{log}}$ ) for all stimulus conditions.

\begin{tabular}{|c|c|c|c|c|c|c|c|c|c|}
\hline & & \multicolumn{8}{|c|}{$\mathrm{BR}$ rate } \\
\hline & & \multicolumn{2}{|c|}{$4 \mathrm{c} / \mathrm{s}, 1.5^{\circ}$} & \multicolumn{2}{|c|}{$8 \mathrm{c} / \mathrm{s}, 1.5^{\circ}$} & \multicolumn{2}{|c|}{$8 \mathrm{c} / \mathrm{s}, 1^{\circ}$} & \multicolumn{2}{|c|}{$8 \mathrm{c} / \mathrm{s}, 0.5^{\circ}$} \\
\hline & & $\rho$ & $p$ & $\rho$ & $p$ & $\rho$ & $p$ & $\rho$ & $p$ \\
\hline \multirow[t]{4}{*}{ MPD } & $4 \mathrm{c} / \mathrm{s}, 1.5^{\circ}$ & 0.17 & 0.30 & 0.14 & 0.40 & 0.13 & 0.43 & 0.10 & 0.54 \\
\hline & $8 \mathrm{c} / \mathrm{s}, 1.5^{\circ}$ & 0.21 & 0.20 & 0.16 & 0.34 & 0.14 & 0.38 & 0.16 & 0.33 \\
\hline & $8 \mathrm{c} / \mathrm{s}, 1^{\circ}$ & 0.18 & 0.27 & 0.08 & 0.62 & 0.06 & 0.69 & 0.08 & 0.61 \\
\hline & $8 \mathrm{c} / \mathrm{s}, 0.5^{\circ}$ & -0.01 & 0.95 & -0.03 & 0.86 & 0.01 & 0.97 & 0.06 & 0.72 \\
\hline
\end{tabular}

\begin{tabular}{|c|c|c|c|c|c|c|c|c|c|}
\hline & & \multicolumn{8}{|c|}{$\mathrm{BR}$ rate } \\
\hline & & \multicolumn{2}{|c|}{$4 \mathrm{c} / \mathrm{s}, 1.5^{\circ}$} & \multicolumn{2}{|c|}{$8 \mathrm{c} / \mathrm{s}, 1.5^{\circ}$} & \multicolumn{2}{|c|}{$8 \mathrm{c} / \mathrm{s}, 1^{\circ}$} & \multicolumn{2}{|c|}{$8 \mathrm{c} / \mathrm{s}, 0.5^{\circ}$} \\
\hline & & $\rho$ & $p$ & $\rho$ & $p$ & $\rho$ & $p$ & $\rho$ & $p$ \\
\hline \multirow[t]{7}{*}{$\mathrm{PR}_{\log }$} & $4 \mathrm{c} / \mathrm{s}, 1.5^{\circ}$ & 0.16 & 0.31 & 0.13 & 0.42 & 0.25 & 0.13 & 0.10 & 0.55 \\
\hline & $8 \mathrm{c} / \mathrm{s}, 1.5^{\circ}$ & -0.06 & 0.71 & 0.06 & 0.71 & 0.00 & 0.99 & -0.13 & 0.42 \\
\hline & $8 \mathrm{c} / \mathrm{s}, 1^{\circ}$ & -0.03 & 0.84 & 0.06 & 0.70 & 0.12 & 0.47 & 0.04 & 0.82 \\
\hline & $8 \mathrm{c} / \mathrm{s}, 0.5^{\circ}$ & -0.16 & 0.32 & -0.21 & 0.20 & -0.13 & 0.43 & -0.16 & 0.34 \\
\hline & & \multicolumn{8}{|c|}{ MPD } \\
\hline & & \multicolumn{2}{|c|}{$4 \mathrm{c} / \mathrm{s}, 1.5^{\circ}$} & \multicolumn{2}{|c|}{$8 \mathrm{c} / \mathrm{s}, 1.5^{\circ}$} & \multicolumn{2}{|c|}{$8 \mathrm{c} / \mathrm{s}, 1^{\circ}$} & \multicolumn{2}{|c|}{$8 \mathrm{c} / \mathrm{s}, 0.5^{\circ}$} \\
\hline & & $\rho$ & $p$ & $\rho$ & $p$ & $\rho$ & $p$ & $\rho$ & $p$ \\
\hline \multirow[t]{4}{*}{$\mathrm{PR}_{\log }$} & $4 \mathrm{c} / \mathrm{s}, 1.5^{\circ}$ & 0.42 & $6.68 \times 10^{-3}$ & 0.35 & $2.68 \times 10^{-2}$ & 0.39 & $1.27 \times 10^{-2}$ & 0.03 & 0.86 \\
\hline & $8 \mathrm{c} / \mathrm{s}, 1.5^{\circ}$ & 0.23 & 0.16 & 0.11 & 0.51 & 0.15 & 0.37 & -0.26 & 0.11 \\
\hline & $8 \mathrm{c} / \mathrm{s}, 1^{\circ}$ & 0.26 & 0.10 & 0.13 & 0.42 & -0.01 & 0.97 & -0.14 & 0.38 \\
\hline & $8 \mathrm{c} / \mathrm{s}, 0.5^{\circ}$ & 0.09 & 0.60 & -0.05 & 0.79 & -0.12 & 0.47 & -0.15 & 0.35 \\
\hline
\end{tabular}

c/s: cycles/s. ${ }^{\circ}$ : degrees of visual angle in a circular aperture. $\rho:$ Spearman's $\rho . p: p$ value (two-tailed).

$1.5^{\circ}$ than a $1^{\circ}$ aperture $\left(p=4.23 \times 10^{-3}\right.$; Bonferroni-adjusted $\alpha$ : $0.05 / 6$ Wilcoxon signed rank tests $=8.33 \times 10^{-3}$; see Table 1 and Fig. 3 b). MPD for the 8 cycles $/ \mathrm{s} 0.5^{\circ}$ stimulus was also significantly longer compared with all other stimuli $\left(p \leq 1.87 \times 10^{-4}\right)$. However, there was no significant difference in MPD between the 4 cycles/s $1.5^{\circ}$ stimulus and 8 cycles/s stimulus in a $1^{\circ}$ or $1.5^{\circ}$ aperture $(p \geq 0.28)$. These comparative results remained non-significant at a less conservative $\alpha$ of 0.05 .

\subsection{Covariance of mixed-percept duration between stimuli}

Spearman's $\rho$ correlations were performed to assess the covariance of individuals' MPDs between all stimulus conditions. There was a pattern of significant, moderate-to-high positive correlations in MPD between all stimulus conditions $\left(\rho=0.51-0.91, \quad p \leq 3.87 \times 10^{-4}\right.$, one-tailed; Bonferroni-adjusted $\alpha$ : $0.05 / 6$ Spearman's $\rho$ tests $=8.33 \times 10^{-3}$ ). This result indicates that individuals' MPDs moderately to strongly covaried across these stimulus conditions, except between the $8 \mathrm{cycles} / \mathrm{s} 0.5^{\circ}$ and 4 cycles $/ \mathrm{s} 1.5^{\circ}$ stimuli ( $p=1.44 \times 10^{-2}$, one-tailed). In addition, a significant high intraclass correlation indicated low variance (or high clustering) in MPD within each stimulus condition ( $r=0.89$, $p=1.26 \times 10^{-21}$; average measures, two-way mixed model). These findings indicate that the observed differences in MPD between the stimulus conditions may be attributed to differences in stimulus parameters, however the findings need to be interpreted with caution due to conflation of the MPD response option with reporting of errors.

\subsection{Predominance}

$\mathrm{PR}_{\log }$ was compared between the stimulus conditions to examine stimulus-strength modulation effects. Normality was violated for the distributions of $\mathrm{PR}_{\log }$ (Shapiro-Wilk test; $p<0.05$ ). A Friedman test with $\mathrm{PR}_{\log }$ as the dependent variable and stimulus conditions as the independent variable showed no significant difference in $\mathrm{PR}_{\log }$ across the stimulus conditions $(p=0.31)$. A one-sample Wilcoxon signed rank test was performed on $\mathrm{PR}_{\log }$ for each stimulus condition to assess the perceptual predilection towards one or the other image. $\mathrm{PR}_{\log }$ was significantly greater than zero indicating perceptual bias towards the vertical grating for the 8 cycles $/ \mathrm{s} 0.5^{\circ}$ stimulus $\left(p=5.97 \times 10^{-3}\right.$; Bonferroni-adjusted $\alpha$ : $0.05 / 4$ Wilcoxon signed rank tests $=1.25 \times 10^{-2}$ ), but not for the remaining stimuli $\left(p \leq 1.55 \times 10^{-2}\right)$.

Spearman's $\rho$ correlations were performed to assess the covariance of individuals' $\mathrm{PR}_{\log }$ between all stimulus conditions. There was a pattern of significant, moderate correlations in $\mathrm{PR}_{\log }$ between all stimulus conditions ( $\rho=0.38-0.66, p \leq 7.32 \times 10^{-3}$, one-tailed; Bonferroniadjusted $\alpha$ : $0.05 / 6$ Spearman's $\rho$ tests $=8.33 \times 10^{-3}$ ). This result indicates that individuals' $\mathrm{PR}_{\log }$ moderately to strongly covaried across the stimulus conditions, except between the 8 cycles $/ \mathrm{s} 0.5^{\circ}$ and $4 \mathrm{cy}-$ cles $/ \mathrm{s} 1.5^{\circ}$ stimuli ( $p=0.11$, one-tailed). In addition, a significant moderate intraclass correlation indicated low variance (or high clustering) in $\mathrm{PR}_{\log }$ within each stimulus condition $(r=0.69$, $p=1.08 \times 10^{-6}$; average measures, two-way mixed model). These findings indicate that individuals' $\mathrm{PR}_{\log }$ values were homogeneously clustered across the stimulus conditions.

\subsection{Association between binocular rivalry measures, psychometric measures and age}

Spearman's $\rho$ correlations were performed between BR rate, MPD and $\mathrm{PR}_{\log }$ for each stimulus condition, to examine the relationship between these measures. No significant correlation was found between BR rate and either MPD or $\mathrm{PR}_{\log }$ across all stimulus conditions $(p \geq 0.30$, two-tailed; Bonferroni-adjusted $\alpha$ : $0.05 / 4$ Spearman's $\rho$ tests $=1.25 \times 10^{-2}$; see Table 2). However, there was a significant, moderate positive correlation between MPD and $\mathrm{PR}_{\log }$ for the 4 cycles/s $1.5^{\circ}$ stimulus $(\rho=0.42$; $\left.p=6.68 \times 10^{-3}\right)$, but not for the remaining stimuli $(p \geq 0.35)$.

The association between each BR measure, psychometric measures 
Table 3

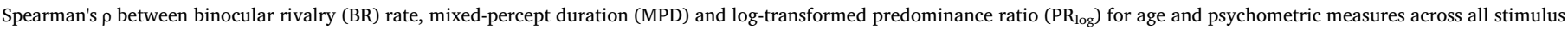
conditions.

\begin{tabular}{|c|c|c|c|c|c|c|c|c|}
\hline & \multicolumn{8}{|c|}{$\mathrm{BR}$ rate } \\
\hline & \multicolumn{2}{|c|}{$4 \mathrm{c} / \mathrm{s}, 1.5^{\circ}$} & \multicolumn{2}{|c|}{$8 \mathrm{c} / \mathrm{s}, 1.5^{\circ}$} & \multicolumn{2}{|c|}{$8 \mathrm{c} / \mathrm{s}, 1^{\circ}$} & \multicolumn{2}{|c|}{$8 \mathrm{c} / \mathrm{s}, 0.5^{\circ}$} \\
\hline & $\rho$ & $p$ & $\rho$ & $p$ & $\rho$ & $p$ & $\rho$ & $p$ \\
\hline $\operatorname{Age}^{\mathrm{a}}$ & -0.16 & 0.16 & -0.30 & $3.25 \times 10^{-2}$ & -0.30 & $2.94 \times 10^{-2}$ & -0.25 & $5.96 \times 10^{-2}$ \\
\hline STAI-state ${ }^{a}$ & 0.16 & 0.16 & 0.14 & 0.20 & 0.11 & 0.25 & 0.09 & 0.29 \\
\hline STAI-trait ${ }^{\mathrm{a}}$ & 0.27 & $4.62 \times 10^{-2}$ & 0.22 & $8.76 \times 10^{-2}$ & 0.16 & 0.17 & 0.15 & 0.18 \\
\hline MÅDRS ${ }^{\mathrm{a}}$ & 0.05 & 0.39 & 0.27 & $4.77 \times 10^{-2}$ & 0.17 & 0.14 & 0.10 & 0.27 \\
\hline Subjective mood & -0.06 & 0.70 & -0.02 & 0.90 & -0.01 & 0.97 & 0.05 & 0.75 \\
\hline
\end{tabular}

\begin{tabular}{|c|c|c|c|c|c|c|c|c|}
\hline & \multicolumn{8}{|c|}{ MPD } \\
\hline & \multicolumn{2}{|c|}{$4 \mathrm{c} / \mathrm{s}, 1.5^{\circ}$} & \multicolumn{2}{|c|}{$8 \mathrm{c} / \mathrm{s}, 1.5^{\circ}$} & \multicolumn{2}{|c|}{$8 \mathrm{c} / \mathrm{s}, 1^{\circ}$} & \multicolumn{2}{|c|}{$8 \mathrm{c} / \mathrm{s}, 0.5^{\circ}$} \\
\hline & $\rho$ & $p$ & $\rho$ & $p$ & $\rho$ & $p$ & $\rho$ & $p$ \\
\hline Age & -0.10 & 0.54 & -0.05 & 0.75 & -0.05 & 0.78 & 0.05 & 0.75 \\
\hline STAI-state & 0.10 & 0.54 & 0.17 & 0.29 & 0.07 & 0.68 & 0.01 & 0.95 \\
\hline STAI-trait & 0.20 & 0.22 & 0.23 & 0.16 & 0.09 & 0.58 & 0.03 & 0.87 \\
\hline MÅDRS & 0.03 & 0.87 & 0.07 & 0.66 & 0.05 & 0.78 & 0.09 & 0.58 \\
\hline \multirow[t]{4}{*}{ Subjective mood } & 0.08 & 0.63 & 0.01 & 0.95 & -0.10 & 0.55 & -0.18 & 0.27 \\
\hline & \multicolumn{8}{|c|}{$\mathrm{PR}_{\log }$} \\
\hline & \multicolumn{2}{|c|}{$4 \mathrm{c} / \mathrm{s}, 1.5^{\circ}$} & \multicolumn{2}{|c|}{$8 \mathrm{c} / \mathrm{s}, 1.5^{\circ}$} & \multicolumn{2}{|c|}{$8 \mathrm{c} / \mathrm{s}, 1^{\circ}$} & \multicolumn{2}{|c|}{$8 \mathrm{c} / \mathrm{s}, 0.5^{\circ}$} \\
\hline & $\rho$ & $p$ & $\rho$ & $p$ & $\rho$ & $p$ & $\rho$ & $p$ \\
\hline Age & -0.08 & 0.64 & 0.01 & 0.93 & -0.17 & 0.30 & 0.10 & 0.54 \\
\hline STAI-state & 0.19 & 0.25 & -0.15 & 0.34 & 0.04 & 0.82 & -0.26 & 0.11 \\
\hline STAI-trait & 0.20 & 0.22 & -0.14 & 0.38 & 0.04 & 0.81 & -0.16 & 0.33 \\
\hline MÅDRS & -0.09 & 0.60 & -0.05 & 0.76 & 0.13 & 0.42 & -0.16 & 0.32 \\
\hline Subjective mood & -0.15 & 0.37 & 0.19 & 0.24 & 0.18 & 0.28 & 0.27 & 0.10 \\
\hline
\end{tabular}

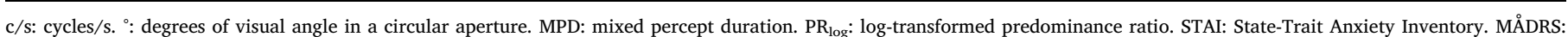
Montgomery-Åsberg Depression Rating Scale. $\rho:$ Spearman's $\rho$. $p$ : $p$ value.

a One-tailed (two-tailed for all other measures).

and age was assessed to examine the relationship between BR and subject factors. Spearman's $\rho$ correlations were performed for age and each of the four psychometric measures (i.e., STAI-state, STAI-trait, MÅDRS, subjective mood rating) to assess their association with BR rate, MPD and $\mathrm{PR}_{\text {log. }}$. No significant correlation was found between age or any psychometric measure with (i) BR rate $\left(p \geq 2.94 \times 10^{-2}\right.$, onetailed; two-tailed for subjective mood; Bonferroni-adjusted $\alpha$ : 0.05/20 Spearman's $\rho$ tests $=2.50 \times 10^{-3}$; see Table 3$)$, (ii) MPD $(p \geq 0.16$, two-tailed) and (iii) $\mathrm{PR}_{\log }$ ( $p \geq 0.10$, two-tailed). At a less conservative $\alpha$ of 0.05 , significant modest correlations were found between BR rate and age, STAI-trait and MÅDS scores for select stimulus conditions $\left(p \leq 4.77 \times 10^{-2}\right)$.

\subsection{Power analysis}

Power analysis indicated that, assuming a power of 0.80 and a conservative Bonferroni-adjusted $\alpha$ of $8.33 \times 10^{-3}$ (0.05/6 Wilcoxon signed rank tests; two-tailed), the current study's sample size of 40 would be sufficient to detect a significant difference in BR rate, MPD and $\mathrm{PR}_{\log }$ between stimulus conditions with a minimum true effect size of 0.60 (Gpower; [23]). Likewise, assuming a power of 0.80 and a conservative Bonferroni-adjusted $\alpha$ of $1.25 \times 10^{-2}(0.05 / 4$ Spearman's $\rho$ tests; two-tailed), the current study's sample size of 40 would be sufficient to detect a significant correlation between BR rate, MPD and $\mathrm{PR}_{\log }$ with a minimum true effect size of 0.49 . For age and psychometric measures, assuming a power of 0.80 and a conservative Bonferroniadjusted $\alpha$ of $2.50 \times 10^{-3}(0.05 / 20$ Spearman's $\rho$ tests), the current study's sample size of 40 would be sufficient to detect a significant correlation between $\mathrm{BR}$ rate and $\mathrm{MPD} / \mathrm{PR}_{\log }$ with a minimum true effect size of 0.52 (one-tailed) and 0.55 (two-tailed), respectively.

\section{Discussion}

The current study represents the largest BR dataset examining the effect of stimulus strength on BR rate, with drift speed as the stimulus strength factor. It is also the largest BR dataset to examine the effect of stimulus aperture size on MPD. Each individual's BR rate and MPD was determined for different grating stimuli that varied in drift speed and aperture size. The current study found that viewing higher-strength stimuli in both eyes (i.e., 8 cycles/s drift speed in $1^{\circ}$ or $1.5^{\circ}$ aperture) induced a significantly faster BR rate. In addition, viewing larger stimuli (i.e., $1.5^{\circ}$ aperture) drifting at either 4 or 8 cycles/s produced a relatively shorter total MPD, supporting the use of a $1.5^{\circ}$ stimulus in generating a more accurate and representative measure of an individual's BR rate. Overall, the findings indicate that of the stimuli assessed in this study, the 8 cycles $/ \mathrm{s} 1.5^{\circ}$ aperture stimulus induced the fastest and most accurate BR rate in healthy individuals.

However, it is important to note that in the current study, the 4 cycles $/ \mathrm{s} 1.5^{\circ}$ aperture stimulus produced a mean $\mathrm{BR}$ rate of $0.53 \mathrm{~Hz}$ (i.e., a switch every $1.89 \mathrm{~s}, n=40$, mean age $=34.4$ years). This finding is comparable to previous studies using this stimulus type in healthy adolescents ([54]; $0.54 \mathrm{~Hz}$ or a switch every $1.85 \mathrm{~s}, n=722$, mean age $=14.1$ years) and healthy adults $([68] ; 0.60 \mathrm{~Hz}$ or a switch every $1.67 \mathrm{~s}, n=49$, age range $=19-55$ years; [82]; $0.53 \mathrm{~Hz}$ or a switch every 
$1.89 \mathrm{~s}, n=24$, mean age $=32.3$ years). In the current study, the mean $\mathrm{BR}$ rate of the 8 cycles/s $1.5^{\circ}$ stimulus (i.e., $0.57 \mathrm{~Hz}$ or a switch every $1.75 \mathrm{~s}$ ), although significantly faster according to statistical analysis, is only marginally different from the rate for the 4 cycles $/ \mathrm{s} 1.5^{\circ}$ stimulus, and only marginally different from the rates for 4 cycles/s $1.5^{\circ}$ stimuli used in previous studies $[54,68,82]$. Although there are only small differences in BR rate between these high-strength drifting BR stimuli in the current study (i.e., 4 and 8 cycles/s), and the high-strength drifting BR stimuli in previous studies, they all induced markedly faster BR rates than those induced by stationary grating stimuli. These stationary stimuli entailed: (i) a $2^{\circ}$ aperture and spatial frequency of 2 cycles $/{ }^{\circ}$ in a recent large sample ([9]; $0.28 \mathrm{~Hz}$ or a switch every $3.57 \mathrm{~s}, n=1051$, age range $=16-40$ years; stimulus luminance of $39 \mathrm{~cd} / \mathrm{m}^{2}$ ), and (ii) a $1.5^{\circ}$ aperture and a spatial frequency of 4 cycles $/{ }^{\circ}([53] ; 0.40 \mathrm{~Hz}$ or a switch every $2.50 \mathrm{~s}, n=30$, age range $=27-63$ years). However, it is worth noting that Bosten et al. [9] did not exclude responses to mixed percepts, which would have the effect of yielding an apparently slower BR rate. Nonetheless, given large BR rate differences between drifting and stationary stimuli used across all these studies - but only small BR rate differences between the two high-strength stimuli used in the current study - it is unlikely that further increasing drift speed above 8 cycles/s will induce faster BR rates with any meaningful relevance for large-scale BR studies (see below).

It is also noteworthy that the spatial frequency of gratings drifting at 4 cycles/s in the current study is lower than that of the same drift speed in previous studies $[54,68]$, yet the resulting BR rate obtained in the current study was similar to that obtained in the previous studies. This observation suggests that the reduction in spatial frequency in the current study to 5.33 cycles $/{ }^{\circ}$ (cf. 8 cycles $/{ }^{\circ}$ in the previous studies) is unlikely to affect BR rate to any great degree. In the current study we did not vary spatial frequency to examine this issue directly. Such studies have been performed however, albeit involving small sample sizes and stationary gratings, showing the sensitivity function for spatial frequency to be non-monotonic and peaking at approximately 4 cycles $/^{\circ}[44,63]$. With respect to all stimulus parameters, it remains possible that additional modulation of BR rate (and MPD) might become evident if a wider range of such parameters (e.g., spatial frequency) and a wider range of values within each parameter (e.g., drift speeds, aperture sizes) were tested than those in the current study. Further work with multiple stimulus parameter combinations and variations could clarify their effect on BR temporal dynamics (e.g., [78]). The implications of the current BR rate findings for future endophenoptype studies are discussed further below.

Returning to MPD, the results also show that increasing the aperture size of an 8 cycles/s stimulus from $1^{\circ}$ to $1.5^{\circ}$ significantly reduced the total MPD in a given observation period, with MPD not differing significantly between 4 and 8 cycles/s stimuli in a $1.5^{\circ}$ aperture. This finding is in contrast to reports that increasing stimulus aperture size decreases exclusive visibility (i.e., increases MPD; $[8,63,78]$ ). However, the stimuli assessed in the current study subtended $0.5^{\circ}, 1^{\circ}$ and $1.5^{\circ}$, thus varying across a much narrower range than that in [63]; i.e., stimuli subtended between $0.5^{\circ}$ and $8^{\circ}$ ). In O'Shea et al. [63], regarding the data for stimulus aperture sizes similar to that used in the current study (i.e., $0.5^{\circ}, 1^{\circ}$ and $2^{\circ}$ in their study cf. $0.5^{\circ}, 1^{\circ}$ and $1.5^{\circ}$ in the current study), as well as spatial frequency similar to that in the current study (i.e., 4 cycles ${ }^{\circ}$ in their study cf. 5.33 cycles $/{ }^{\circ}$ in the current study), it was evident from their two subjects for whom data were reported in full, that MPD (by way of assessing exclusive percept visibility) exhibited either of the following patterns: (i) it increased with aperture size in one subject (in contrast to the present findings); or (ii) it increased minimally with aperture size in the other subject. Importantly, the current study used a much larger sample size of 40 than the few subjects used in earlier studies ([8,63,78]; $n=3$ and 4 and 11 , respectively). It should also be noted that the current study used drifting gratings rather than stationary gratings used in those earlier studies. One way to further probe these discrepant findings therefore, is to repeat the current experiment using both drifting and stationary stimuli in a large sample of healthy individuals.

It is also important to note that the MPD findings of the current study should be interpreted with caution due to conflation of the MPD response option with reporting of erroneous responses. This response protocol raises the possibility that rather than MPD per se varying according to stimulus size, it may be that the erroneous response rate varies in this way. That is, a higher erroneous response rate for the smallest aperture size could also explain the apparent MPD finding for that size. Even if this scenario was the case however, it would still be true that the smallest aperture size yielded a less accurate measure of an individual's true $\mathrm{BR}$ rate (be it through extra errors with this size, a longer MPD, or both of these factors).

Regarding other measures collected in the current study, it was found that although there was a significant perceptual bias towards the left-eye's presented image for all but one of the stimuli (i.e., 8 cycles $/ \mathrm{s}$ $0.5^{\circ}$ stimulus), this predominance was not significantly correlated with $\mathrm{BR}$ rate (i.e., predominance can vary while BR rate remains constant). Individuals' $\mathrm{BR}$ rates and MPDs were also not associated with each other. In addition, each BR measure (BR rate, MPD, $\mathrm{PR}_{\log }$ ) was not associated with age, state anxiety, trait anxiety, depressive state, and subjective mood, for any stimulus condition. The present findings are consistent with reports of no significant association between BR rate and state anxiety [5]. However, the findings conflict with reports that slower BR rate is associated with increasing age $[9,38,81]$ and lower trait anxiety ([5]; though noting the low correlation in that study; see also [56]). Nonetheless the results from the current study are in the same direction as these previously reported findings.

Potential explanations for these discrepant results are worth mentioning. For example, in regard to BR rate, it is possible that more statistical power from a larger sample size could reveal significant correlations with age and psychometric measures. In addition, the experimental protocol of the current study enabled stabilization of an individual's BR rate, which was not the case in other studies that examined BR rate in relation to age $[9,38,81]$. The current study also determined BR rate using longer observation periods of seven minutes (post-stabilization). This protocol difference meant that a greater amount of individual BR data was collected in the current study compared with previous studies showing the relationship between BR rate and age/anxiety (except [56], in which the same amount of data was collected). It is also worth noting that the complexity of BR stimuli was different between the current study and that of [5]; i.e., orthogonal gratings cf. house-face images, respectively).

In addition to having implications for large-scale studies of BR rate as a $\mathrm{BD}$ endophenotype, the present main finding of higher strength stimuli in both eyes inducing faster BR rate confirms Levelt's [48] Proposition IV of BR dynamics for the first time using drift speed as the stimulus strength factor. An interesting additional question regarding Levelt's Proposition IV is whether individual variation in (matched-eye) stimulus-related modulation of BR rate is exhibited. The current study found that a majority of healthy individuals reported stimulus-related modulation of BR rate consistent with Levelt's Proposition IV, but also that a sizable proportion of the large sample $(n=11$ of 40$)$ reported the reverse effect (i.e., a slower BR rate with greater stimulus strength presented to both eyes). This individual variation observation underscores the importance of attending to individual variation in psychophysical BR studies - by using larger sample sizes than those traditionally used - as well as in other visual, sensory, behavioral and computational neuroscience studies.

Investigation of slow $\mathrm{BR}$ rate as an endophenotype for $\mathrm{BD}$, and of $\mathrm{BR}$ anomalies in other clinical conditions, requires large-scale studies, standardized test protocols, and optimal stimuli for maximal group separation. The current study has shown that high-strength $1.5^{\circ}$ aperture BR stimuli with 8 cycles/s drift speed induced a significantly faster and more accurate BR rate in healthy individuals. However, the findings also show that BR stimuli drifting at 4 cycles/s, as used in previous 
studies (see above), induce BR rates only minimally slower than stimuli drifting at 8 cycles/s. For this reason, it cannot yet be recommended to change to the faster drift speed for endophenotype studies until there has been direct assessment of this stimulus strength issue in a BD cohort.

As further research emerges on important issues of stimulus optimization and BR test platforms suitable for very large-scale studies, the field may also move towards standardized BR testing protocols across clinical conditions and research centres. Achieving this goal will enable comparisons to be made between clinical BR studies and thereby shed light on commonalities and differences between underlying pathophysiologies. Explicitly addressing issues of individual differences in BR dynamics may also provide clues to understanding the neurobiology of both BR and its anomalies across a spectrum of brain disorders.

\section{Disclosures}

The authors of this paper do not have any commercial associations that might pose a conflict of interest in connection with this manuscript.

\section{Acknowledgements}

We thank all volunteers who participated in the study and an anonymous reviewer for helpful comments on an earlier version of this manuscript. TTN was supported by National Health and Medical Research Council [grant number 490976]. SMM received support from National Health and Medical Research Council, Defence Health Foundation, a 2012 NARSAD Young Investigator Grant from Brain \& Behavior Research Foundation, USA) [grant number 19163], and Monash Institute of Medical Engineering.

\section{References}

[1] M. Aafjes, J.E. Heuting, P. Visser, Individual and interindividual differences in binocular retinal rivalry in man, Psychophysiology 3 (1966) 18-22, http://dx.doi. org/10.1111/j.1469-8986.1966.tb02674.x.

[2] L.T. Alexander, P.D. Bricker, Figure-ground contrast and binocular rivalry, J. Exp. Psychol. 44 (1952) 452-454, http://dx.doi.org/10.1037/h0053965.

[3] J.A. Amador-Campos, J.A. Aznar-Casanova, M. Moreno-Sánchez, A. Medina-Peña, J.J. Ortiz-Guerra, Psychometric properties of a test for ADHD based on binocular rivalry, Span. J. Psychol. 16 (2013) 1-8, http://dx.doi.org/10.1017/sjp.2013.34.

[4] J.A. Amador-Campos, J.A. Aznar-Casanova, J.J. Ortiz-Guerra, M. Moreno-Sánchez, A. Medina-Peña, Assessing attention deficit by binocular rivalry, J. Atten. Disord. 19 (2015) 1064-1073, http://dx.doi.org/10.1177/1087054713482686.

[5] E.C. Anderson, M.T. Dryman, J. Worthington, E.A. Hoge, L.E. Fischer, M.H. Pollack, ... N.M. Simon, Smiles may go unseen in generalized social anxiety disorder: evidence from binocular rivalry for reduced visual consciousness of positive facial expressions, J. Anxiety Disord. 27 (2013) 619-626, http://dx.doi.org/10.1016/j. janxdis.2013.07.004.

[6] J.A. Aznar Casanova, J.A. Amador Campos, M. Moreno Sánchez, H. Supèr, Onset time of binocular rivalry and duration of inter-dominance periods as psychophysical markers of ADHD, Perception 42 (2013) 16-27, http://dx.doi.org/10.1068/p7203.

[7] E.H. Bárány, U. Halldén, The influence of some central nervous depressants on the reciprocal inhibition between the two retinae as manifested in retinal rivalry, Acta Physiol. Scand. 13 (1947) 296-316, http://dx.doi.org/10.1111/j.1748-1716.1947. tb00427.x.

[8] R. Blake, R.P. O'Shea, T.J. Mueller, Spatial zones of binocular rivalry in central and peripheral vision, Vis. Neurosci. 8 (1992) 469-478, http://dx.doi.org/10.1017/ S0952523800004971.

[9] J.M. Bosten, P.T. Goodbourn, A.J. Lawrance-Owen, G. Bargary, R.E. Hogg, J.D. Mollon, A population study of binocular function, Vis. Res. 110 (2015) 34-50, http://dx.doi.org/10.1016/j.visres.2015.02.017.

[10] D.H. Brainard, The psychophysics toolbox, Spat. Vis. 10 (1997) 433-436, http://dx. doi.org/10.1163/156856897X00357.

[11] J.W. Brascamp, P.C. Klink, W.J.M. Levelt, The 'laws' of binocular rivalry: 50 years of Levelt's propositions, Vis. Res. 109 (2015) 20-37, http://dx.doi.org/10.1016/j. visres.2015.02.019.

[12] B.B. Breese, On inhibition, Psychol. Rev. Monogr. Suppl. 3 (1899) 1-65, http://dx. doi.org/10.1037/h0092990.

[13] B.B. Breese, Binocular rivalry, Psychol. Rev. 16 (1909) 410-415, http://dx.doi.org/ $10.1037 / \mathrm{h} 0075805$

[14] D.E. Cameron, Studies in depression, J. Ment. Sci. 82 (1936) 148-161, http://dx. doi.org/10.1192/bjp.82.337.148.

[15] C. Cappe, A. Clarke, C. Mohr, M.H. Herzog, Is there a common factor for vision? J. Vis. 14 (2014) 1-11, http://dx.doi.org/10.1167/14.8.4.
[16] R. Cogan, A.G. Goldstein, Reporting of fragmentations in the binocular rivalry of contours, Am. J. Psychol. 85 (1972) 569-584, http://dx.doi.org/10.2307/1421719.

[17] G. D'Agata, G. Gaffuri, La percezione del cambiamento di prospettiva di una figura ambigua nella schizophrenia, Neuropsichiatrica 2 (1968) 357-363.

[18] M. Donnelly, R.J. Miller, Ingested ethanol and binocular rivalry, Invest. Ophthalmol. Vis. Sci. 36 (1995) 1548-1554.

[19] R. van Ee, Stochastic variations in sensory awareness are driven by noisy neuronal adaptation: evidence from serial correlations in perceptual bistability, J. Opt. Soc. Am. A 26 (2009) 2612-2622, http://dx.doi.org/10.1364/JOSAA.26.002612.

[20] J.H. Ewen, The psychological estimation of the effects of certain drugs upon the syntonic and schizophrenic psychoses: with a brief inquiry into a physiological basis of temperament, J. Ment. Sci. 77 (1931) 742-766, http://dx.doi.org/10.1192/bjp. 77.319.742.

[21] H.J. Eysenck, Cyclothymia and schizothymia as a dimension of personality, Exp. J. Personal. 20 (1952) 345-384, http://dx.doi.org/10.1111/j.1467-6494.1952. tb01115.x.

[22] M. Fahle, Binocular rivalry: suppression depends on orientation and spatial frequency, Vis. Res. 22 (1982) 787-800, http://dx.doi.org/10.1016/0042-6989(82) 90010-4.

[23] F. Faul, E. Erfelder, GPower: A Priori-, Post Hoc-, and Compromise Power Analyses for MS-DOS [Computer Program], Bonn University, Bonn, Germany, 1992.

[24] J. Flint, M.R. Munafò, The endophenotype concept in psychiatric genetics, Psychol. Med. 37 (2007) 163-180, http://dx.doi.org/10.1017/S0033291706008750.

[25] R. Fox, Rate of binocular rivalry alternation in psychotic and nonpsychotic patients, J. Abnorm. Psychol. 70 (1965) 34-37, http://dx.doi.org/10.1037/h0021684.

[26] J. Freyberg, C.E. Robertson, S. Baron-Cohen, Reduced perceptual exclusivity during object and grating rivalry in autism, J. Vis. 15 (2015) 1-12, http://dx.doi.org/10 $1167 / 15.13 .11$

[27] A.S. Friedman, Minimal effects of severe depression on cognitive functioning, J. Abnorm. Soc. Psychol. 69 (1964) 237-243, http://dx.doi.org/10.1037/h0048608.

[28] R.W. George, The significance of the fluctuations experienced in observing ambiguous figures and in binocular rivalry, J. Gen. Psychol. 15 (1936) 39-61, http:// dx.doi.org/10.1080/00221309.1936.9917904.

[29] A.G. Goldstein, Retinal rivalry and Troxler's effect: a correlation, Percept. Psychophys. 4 (1968) 261-263, http://dx.doi.org/10.3758/BF03210511.

[30] I.I. Gottesman, T.D. Gould, The endophenotype concept in psychiatry: etymology and strategic intentions, Am. J. Psychiatry 160 (2003) 636-645, http://dx.doi.org/ 10.1176/appi.ajp.160.4.636.

[31] T.D. Gould, I.I. Gottesman, Psychiatric endophenotypes and the development of valid animal models, Genes Brain Behav. 5 (2006) 113-119, http://dx.doi.org/10. 1111/j.1601-183X.2005.00186.x.

[32] L. Grzeczkowski, A.M. Clarke, G. Fancis, F.W. Mast, M.H. Herzog, About individual differences in vision, Vis. Res. (2017), http://dx.doi.org/10.1016/j.visres.2016.10. 006.

[33] G. Hasler, W.C. Drevets, T.D. Gould, I.I. Gottesman, H.K. Manji, Toward constructing an endophenotype strategy for bipolar disorders, Biol. Psychiatry 60 (2006) 93-105, http://dx.doi.org/10.1016/j.biopsych. 2005.11.006.

[34] W.F. Hodges, R. Fox, Effect of arousal and intelligence on binocular rivalry rate Percept. Mot. Skills 20 (1965) 71-75.

[35] M. Hollins, The effect of contrast on the completeness of binocular rivalry suppression, Percept. Psychophys. 27 (1980) 550-556, http://dx.doi.org/10.3758/ BF03198684.

[36] E.P. Hong, J.W. Park, Sample size and statistical power calculation in genetic association studies, Genom. Infom. 10 (2012) 117-122, http://dx.doi.org/10.5808/ GI.2012.10.2.117.

[37] J. Hunt, J.P. Guilford, Fluctuation of an ambiguous figure in dementia praecox and in manic-depressive patients, J. Abnorm. Soc. Psychol. 27 (1933) 443-452, http:// dx.doi.org/10.1037/h0071060.

[38] E. Jalavisto, The phenomenon of retinal rivalry in the aged, Gerontol. Clin. 9 (1964) 1-8, http://dx.doi.org/10.1159/000211230.

[39] T. Jia, X. Ye, Q. Wei, W. Xie, C. Cai, J. Mu, ... K. Wang, Difference in the binocular rivalry rate between depressive episodes and remission, Physiol. Behav. 151 (2015) 272-278, http://dx.doi.org/10.1016/j.physbeh.2015.08.007.

[40] R. Kanai, G. Rees, The structural basis of interindividual differences in human behaviour and cognition, Nat. Rev. Neurosci. 12 (2011) 231-242, http://dx.doi.org/ 10.1038/nrn3000.

[41] R. Kanai, B. Bahrami, G. Rees, Human parietal cortex structure predicts individual differences in perceptual rivalry, Curr. Biol. 20 (2010) 1626-1630, http://dx.doi. org/10.1016/j.cub.2010.07.027.

[42] T. Karaminis, C. Lunghi, L. Neil, D. Burr, E. Pellicano, Binocular rivalry in children on the autism spectrum, Autism Res. 10 (2017) 1096-1106, http://dx.doi.org/10. 1002/aur.1749.

[43] K.S. Kendler, M.C. Neale, Endophenotype: a conceptual analysis, Mol. Psychiatry 15 (2010) 789-797, http://dx.doi.org/10.1038/mp.2010.8.

[44] F.L. Kitterle, J. Thomas, The effects of spatial frequency, orientation, and color upon binocular rivalry and monocular pattern alternation, Bull. Psychon. Soc. 16 (1980) 405-407, http://dx.doi.org/10.3758/BF03329581.

[45] R.J. Klein, Power analysis for genome-wide association studies, BMC Genet. 8 (2007) 117-122, http://dx.doi.org/10.1186/1471-2156-8-58.

[46] P.C.F. Law, B.K. Paton, R.H. Thomson, G.B. Liu, S.M. Miller, T.T. Ngo, Dichoptic viewing methods for binocular rivalry research: prospects for large-scale clinical and genetic studies, Twin Res. Hum. Genet. 16 (2013) 1033-1078, http://dx.doi. org/10.1017/thg.2013.76.

[47] P.C.F. Law, B.K. Paton, J.A. Riddiford, C.T. Gurvich, T.T. Ngo, S.M. Miller, No relationship between binocular rivalry rate and eye-movement profiles in healthy individuals: a Bayes factor analysis, Perception 44 (2015) 643-661, http://dx.doi. 
org $/ 10.1177 / 0301006615594267$.

[48] W. Levelt, On binocular rivalry, Institute for Perception RVO-TNO, Soesterberg, The Netherlands, 1965.

[49] A.M. van Loon, T. Knapen, H.S. Scholte, E. St. John-Saaltink, T.H. Donner, V.A.F. Lamme, GABA shapes the dynamics of bistable perception, Curr. Biol. 23 (2013) 823-827, http://dx.doi.org/10.1016/j.cub.2013.03.067.

[50] N.G. Martin, L.J. Eaves, M.J. Kearsey, P. Davies, The power of the classical twin study, Heredity 40 (1977) 97-116, http://dx.doi.org/10.1038/hdy.1977.9.

[51] W. McDougall, Outline of Abnormal Psychology, Charles Scribner's Sons, New York, 1926, http://dx.doi.org/10.1086/207638.

[52] W. McDougall, M. Smith, The Effects of Alcohol and Some Other Drugs during Normal and Fatigued Conditions, HMS Office, London, 1920.

[53] S.M. Miller, B.D. Gynther, K.R. Heslop, G.B. Liu, P.B. Mitchell, T.T. Ngo, .. L.B. Geffen, Slow binocular rivalry in bipolar disorder, Psychol. Med. 33 (2003) 683-692, http://dx.doi.org/10.1017/S0033291703007475.

[54] S.M. Miller, N.K. Hansell, T.T. Ngo, G.B. Liu, J.D. Pettigrew, N.G. Martin, M.J. Wright, Genetic contribution to individual variation in binocular rivalry rate, Proc. Natl. Acad. Sci. USA 107 (2010) 2664-2668, http://dx.doi.org/10.1073/pnas. 0912149107.

[55] S.A. Montgomery, M. Åsberg, A new depression scale designed to be sensitive to change, Br. J. Psychiatry 134 (1979) 382-389, http://dx.doi.org/10.1192/bjp.134. 4.382.

[56] M. Nagamine, A. Yoshino, M. Yamazaki, M. Obara, S. Sato, Y. Takahashi, S. Nomura, Accelerated binocular rivalry with anxious personality, Physiol. Behav. 91 (2007) 161-165, http://dx.doi.org/10.1016/j.physbeh.2007.02.016.

[57] M. Nagamine, A. Yoshino, M. Miyazaki, Y. Takahashi, S. Nomura, Difference in binocular rivalry rate between patients with bipolar I and bipolar II disorders, Bipolar Disord. 11 (2009) 539-546, http://dx.doi.org/10.1111/j.1399-5618.2009. 00719.x.

[58] N. Nemor, The alternation of an ambiguous figure in paretics and schizophrenics, J. Abnorm. Soc. Psychol. 48 (1953) 445-447, http://dx.doi.org/10.1037/h0055080.

[59] T.T. Ngo, P.B. Mitchell, N.G. Martin, S.M. Miller, Psychiatric and genetic studies of binocular rivalry: an endophenotype for bipolar disorder? Acta Neuropsychiatr. 23 (2011) 37-42, http://dx.doi.org/10.1111/j.1601-5215.2010.00510.x.

[60] T.T. Ngo, W.N. Barsdell, P.C.F. Law, S.M. Miller, Binocular rivalry, brain stimulation and bipolar disorder, in: S.M. Miller (Ed.), The Constitution of Visual Consciousness: Lessons from Binocular Rivalry, vol. 90, John Benjamins Publishing Company, Amsterdam, The Netherlands, 2013, pp. 211-252, , http://dx.doi.org/ 10.1075/aicr.90.09ngo.

[61] H.F. Norman, J.F. Norman, J. Bilotta, The temporal course of suppression during binocular rivalry, Perception 29 (2000) 831-841, http://dx.doi.org/10.1068/ p3085.

[62] R.C. Oldfield, The assessment and analysis of handedness: the Edinburgh inventory, Neuropsychologia 9 (1971) 97-113, http://dx.doi.org/10.1016/0028-3932(71) 90067-4.

[63] R.P. O'Shea, A.J.H. Sims, D.G. Govan, The effect of spatial frequency and field size on the spread of exclusive visibility in binocular rivalry, Vis. Res. 37 (1997) 175-183, http://dx.doi.org/10.1016/S0042-6989(96)00113-7.

[64] R. O'Shea, A. Parker, D. Alais, Monocular rivalry exhibits three hallmarks of binocular rivalry: evidence for common processes, Vis. Res. 49 (2009) 671-681, http:// dx.doi.org/10.1016/j.visres.2009.01.020.

[65] V. Patel, S. Stuit, R. Blake, Individual differences in temporal dynamics of binocular rivalry and of stimulus rivalry, Psychon. Bull. Rev. 22 (2015) 476-482, http://dx. doi.org/10.3758/s13423-014-0695-1.

[66] D.G. Pelli, The VideoToolbox software for visual psychophysics: transforming numbers into movies, Spat. Vis. 10 (1997) 437-442, http://dx.doi.org/10.1163/ $156856897 X 00366$.

[67] D.H. Peterzell, Discovering sensory processes using individual differences: a review and factor analytic manifesto, Electronic Imaging, Human Vision and Electronic Imaging, 2016, pp. 1-11, , http://dx.doi.org/10.2352/ISSN.2470-1173.2016. 16HVEI-112.

[68] J.D. Pettigrew, S.M. Miller, A ‘sticky’ interhemispheric switch in bipolar disorder?
Proc. R. Soc. Lond. B Biol. Sci. 265 (1998) 2141-2148, http://dx.doi.org/10.1098/ rspb.1998.0551.

[69] A. Platonov, J. Goossens, Influence of contrast and coherence on the temporal dynamics of binocular motion rivalry, PLoS One 8 (2013) 1-12, http://dx.doi.org/10 1371/journal.pone.0071931.

[70] R Core Team, R: A Language and Environment for Statistical Computing [Computer Program], (2016) (Vienna, Austria).

[71] C.E. Robertson, D.J. Kravitz, J. Freyberg, S. Baron-Cohen, C.I. Baker, Slower rate of binocular rivalry in autism, J. Neurosci. 33 (2013) 16983-16991, http://dx.doi. org/10.1523/jneurosci.0448-13.2013.

[72] C.E. Robertson, E.-M. Ratai, N. Kanwisher, Reduced GABAergic action in the autistic brain, Curr. Biol. 26 (2016) 80-85, http://dx.doi.org/10.1016/j.cub.2015.11. 019.

[73] C.P. Said, R.D. Egan, N.J. Minshew, M. Behrmann, D.J. Heeger, Normal binocular rivalry in autism: implications for the excitation/inhibition imbalance hypothesis, Vis. Res. 77 (2013) 59-66, http://dx.doi.org/10.1016/j.visres.2012.11.002.

[74] B.R. Sappenfield, R.J. Ripke, Validities of three visual tests for differentiating organics from schizophrenics and normals, J. Clin. Psychol. 17 (1961) 276-278, http://dx.doi.org/10.1002/1097-4679(196107)17:3<276:AID JCLP2270170316 > 3.0.CO;2-N.

[75] H.H. Seedorff, Effect of alcohol on the motor fusion reserves and stereopsis as well as on the tendency to nystagmus, Acta Ophthalmol. 34 (1956) 273-280, http://dx. doi.org/10.1111/j.1755-3768.1956.tb03361.x

[76] R.W. Shannon, C.J. Patrick, Y. Jiang, E. Bernat, S. He, Genes contribute to the switching dynamics of bistable perception, J. Vis. 11 (2011) 1-7, http://dx.doi.org/ 10.1167/11.3.8.

[77] D.V. Sheehan, Y. Lecrubier, K.H. Sheehan, P. Amorim, J. Janavs, E. Weiller, .. G. Dunbar, The Mini International Neuropsychiatric Interview (M.I.N.I.): the development and validation of a structured diagnostic psychiatric interview, J. Clin. Psychiatry 59 (1998) 22-33.

[78] J. Skerswetat, M.A. Formankiewicz, S.J. Waugh, Very few exclusive percepts for contrast-modulated stimuli during binocular rivalry, Vis. Res. 121 (2016) 10-22, http://dx.doi.org/10.1016/j.visres.2016.01.002.

[79] C.D. Spielberger, R.L. Gorsuch, R. Lushene, P.R. Vagg, G.A. Jacobs, Manual for the State-Trait Anxiety Inventory, Consulting Psychologists Press, Palo Alto, CA, 1983.

[80] S. Suzuki, M. Grabowecky, Long-term speeding in perceptual switches mediated by attention-dependent plasticity in cortical visual processing, Neuron 56 (2007) 741-753, http://dx.doi.org/10.1016/j.neuron.2007.09.028.

[81] K. Ukai, H. Ando, J. Kuze, Binocular rivalry alternation rate declines with age, Percept. Mot. Skills 97 (2003) 393-397, http://dx.doi.org/10.2466/pms.2003.97.2. 393.

[82] E. Vierck, R.J. Porter, S.E. Luty, S. Moor, M.T. Crowe, J.D. Carter, ... P.R. Joyce Further evidence for slow binocular rivalry rate as a trait marker for bipolar disorder, Aust. N. Z. J. Psychiatry 47 (2013) 371-379, http://dx.doi.org/10.1177/ 0004867412474105.

[83] N.J. Wade, C.M.M. de Weert, M.T. Swanston, Binocular rivalry with moving patterns, Percept. Psychophys. 35 (1984) 111-122, http://dx.doi.org/10.3758/ BF03203891.

[84] N.J. Wade, T.T. Ngo, Early views on binocular rivalry, in: S.M. Miller (Ed.), The Constitution of Visual Consciousness: Lessons from Binocular Rivalry, vol. 90, John Benjamins Publishing Company, Amsterdam, The Netherlands, 2013, pp. 77-108, http://dx.doi.org/10.1075/aicr.90.04wad.

[85] M. Wexler, M. Duyck, P. Mamassian, Persistent states in vision break universality and time invariance, Proc. Natl. Acad. Sci. USA 112 (2015) 14990-14995, http:// dx.doi.org/10.1073/pnas.1508847112.

[86] N.R. Wray, S.H. Lee, D. Mehta, A.A.E. Vinkhuyzen, F. Dudbridge, C.M. Middeldorp, Research review: polygenic methods and their application to psychiatric traits, J. Child Psychol. Psychiatry 55 (2014) 1068-1087, http://dx.doi.org/10.1111/jcpp. 12295.

[87] R. Zhu, X. Ye, X. Zhou, J. Yang, Y. Yue, K. Wang, Applications of the binocular rivalry paradigm in patients with bipolar disorder, Chin. J. Nerv. Ment. Dis. 8 (2013) 449-452, http://dx.doi.org/10.3936/j.issn.1002-0152.2013.08.001. 